\title{
ON THE SPECTRUM OF THE NEGATIVE LAPLACIAN FOR GENERAL DOUBLY-CONNECTED BOUNDED DOMAINS
}

\author{
E. M. E. ZAYED and A. I. YOUNIS \\ Mathematics Department, Faculty of Science \\ Zagazig University, Zagazig, Egypt
}

(Received June 17, 1993 and in revised form June 6, 1994)

\begin{abstract}
This paper is devoted to asymptotic formulas for functions related with the spectrum of the standard Laplace operator in two and three dimensional bounded doubly connected domains with impedance boundary conditions, where the impedances are assumed to be positive functions. Moreover, asymptotic expressions for the difference of eigenvalues related to impedance boundary value problems with different impedances are derived. Further results may be obtained.
\end{abstract}

KEY WORDS AND PHRASES: Eigenvalues of the negative Laplacian, doubly connected domains, impedance eigenvalue problem, asymptotic expansions of the heat kernel.

\section{AMS SUBJECT CLASSIFICATION CODES: 35K, 35P.}

\section{INTRODUCTION}

The underlying problem is to deduce the geometrical properties of a membrane from a complete knowledge of the eigenvalues $\left\{\mu_{k}(\sigma)\right\}_{k=1}^{\infty}$ for the negative Laplacian $-\Delta_{n}=-\sum_{i=1}^{n}\left(\frac{\partial}{\partial x^{2}}\right)^{2}$ in $R^{n}, n=2$ or 3.

Let $\Omega$ be a simply connected bounded domain in $R^{n}$ with a smooth boundary $\partial \Omega$ in the case $n=2$, or a smooth bounding surface $S$ in the case $n=3$. Consider the impedance problem

$$
\begin{gathered}
\left(\Delta_{n}+\lambda\right) u=0 \text { in } \Omega, \\
\left(\frac{\partial}{\partial n}+\sigma\right) u=0 \text { on } \partial \Omega(\text { or } S),
\end{gathered}
$$

where $\frac{\partial}{\partial n}$ denotes differentiation along the inward-pointing normal to $\partial \Omega$ (or $S$ ), and $\sigma$ is a positive function. Denote its eigenvalues, counted according to multiplicity, by

$$
0<\mu_{1}(\sigma) \leq u_{2}(\sigma) \leq \ldots \leq \mu_{k}(\sigma) \leq \ldots \rightarrow \infty \text { as } k \rightarrow \infty .
$$

At the beginning of this century the principal problem was that of investigating the asymptotic distribution of the eigenvalues (1.3). It is well known [1] that in the case $\boldsymbol{n}=\mathbf{2}$

$$
\mu_{k}(\sigma) \sim\left(\frac{4 \pi}{|\Omega|}\right) k \quad \text { as } k \rightarrow \infty,
$$

while in the case $n=3$

$$
\mu_{k}(\sigma) \sim\left(\frac{6 \pi^{2}}{V} k\right)^{2 / 3} \text { as } k \rightarrow \infty,
$$

where $|\Omega|$ and $V$ are respectively the area and the volume of the domain $\Omega$. The problem of determining further information about the geometry of $\Omega$ has been discussed by many authors, see 
for example Pleijel [2,3], Kac [4], McKean and Singer [5], Stewartson and Waechter [6], Waechter [7], Greiner [8], Smith [9], Gottlieb [10-12], Hsu [13], Sleeman and Zayed [14,15] and Zayed [16-23], using the asymptotic behavior of the spectral function

$$
\Theta(t)=\sum_{k=1}^{\infty} \exp \left[-t \mu_{k}(\sigma)\right] \text { as } t \rightarrow 0 .
$$

Thus, if $\sigma=0$ (Neumann problem), it is well known that in the case $n=2$

$$
\Theta(t)=\frac{|\Omega|}{4 \pi t}+\frac{|\partial \Omega|}{8(\pi t)^{1 / 2}}+a_{0}+\frac{7}{256}\left(\frac{t}{\pi}\right)^{1 / 2} \int_{\partial \Omega} K^{2}(Q) d Q+0(t) \text { as } t \rightarrow 0,
$$

while in the case $n=3$

$\Theta(t)=\frac{V}{(4 \pi t)^{3 / 2}}+\frac{|S|}{16 \pi t}+\frac{1}{12 \pi^{3 / 2} t^{1} / 2} \int_{S} H(Q) d Q+\frac{7}{128 \pi} \int_{S}\left[H^{2}(Q)-N(Q)\right] d Q+0\left(t^{1 / 2}\right)$ as $t \rightarrow 0$.

If $\sigma=\infty$ (Dirichlet problem), it is well known that in the case $n=2$

$$
\Theta(t)=\frac{|\Omega|}{4 \pi t}-\frac{|\partial \Omega|}{8(\pi t)^{1 / 2}}+a_{0}+\frac{1}{256}\left(\frac{t}{\pi}\right)^{1 / 2} \int_{\partial \Omega} K^{2}(Q) d Q+0(t) \text { as } t \rightarrow 0,
$$

while in the case $n=3$

$\Theta(t)=\frac{V}{(4 \pi t)^{3 / 2}}-\frac{|S|}{16 \pi t}+\frac{1}{12 \pi^{3 / 2} t^{1} / 2} \int_{S} H(Q) d Q+\frac{1}{128 \pi} \int_{S}\left[H^{2}(Q)-N(Q)\right] d Q+0\left(t^{1 / 2}\right)$ as $t \rightarrow 0$.

An examination of the results (1.7) and (1.9) shows that in the case $n=2$ the first term of $\Theta(t)$ determines the area $|\Omega|$ of $\Omega$, the second term determines the total length $|\partial \Omega|$ of the boundary $\partial \Omega$ and the fourth term determines the curvature $K(Q)$ of $\partial \Omega$ at the point $Q \varepsilon \partial \Omega$ while the sign \pm of the second term determines whether we have a Neumann or a Dirichlet problem. The third term $a_{0}$ in (1.7) and (1.9) has geometric significance, e.g., if $\Omega$ is smooth and convex, then $a_{0}=\frac{1}{6}$ while if $\Omega$ is permitted to have a finite number of smooth convex holes " $h$ ", then $a_{0}=(1-h) \frac{1}{6}$.

Similarly, an examination of the results (1.8) and (1.10) shows that in the case $\boldsymbol{n}=3$ the first term of $\Theta(t)$ determines the volume $V$ of $\Omega$, the second term determines the surface area $|S|$ of $S$, the third term determines the mean curvature $H(Q)=\frac{1}{2}\left[\frac{1}{R_{1}(Q)}+\frac{1}{R_{2}(Q)}\right]$ and the fourth term determines the Gaussian curvature $N(Q)=\frac{1}{R_{1}(Q) R_{2}(Q)}$ of the surface $S$ at the point $Q \varepsilon S$, where $R_{1}$ and $R_{2}$ are the principal radii of curvature, while the sign \pm of the second term of $\Theta(t)$ determines whether we have a Neumann or a Dirichlet problem.

We merely note that aspects of the question of Kac, namely, "Can one hear the shape of a drum?" have been discussed by Sleeman and Zayed [14] when $n=2$ and by Zayed [16] when $n=3$ for problem (1.1)-(1.2) in the case $\sigma$ is a positive constant.

Suppose that $\Omega$ is a general doubly connected bounded domain in $R^{n}, n=2$ or 3 consisting of a simply connected bounded inner domain $\Omega_{1}$ with a smooth boundary $\partial \Omega_{1}$ in the case $n=2$ (or a smooth bounding surface $S_{1}$ in the case $n=3$ ) and a simply connected bounded outer domain $\Omega_{2} \supset \bar{\Omega}_{1}$ with a smooth boundary $\partial \Omega_{2}$ in the case $n=2$ (or a smooth bounding surface $S_{2}$ in the case $n=3$ ). Consider the impedance problem

$$
\begin{gathered}
\left(\Delta_{n}+\lambda\right) u=0 \text { in } \Omega, \\
\left(\frac{\partial}{\partial n_{1}}+\sigma_{1}\right) u=0 \quad \text { on } \partial \Omega_{1}\left(\text { or } S_{1}\right),
\end{gathered}
$$

and 


$$
\left(\frac{\partial}{\partial n_{2}}+\sigma_{2}\right) u=0 \text { on } \partial \Omega_{2}\left(\text { or } S_{2}\right),
$$

where $\frac{\partial}{\partial n_{1}}$ and $\frac{\partial}{\partial n_{2}}$ denote differentiations along the inward-pointing normals to $\partial \Omega_{1}\left(\right.$ or $\left.S_{1}\right)$ and $\partial \Omega_{2}$ (or $S_{2}$ ) respectively, in which the impedances $\sigma_{1}$ and $\sigma_{2}$ are positive functions.

Denote its eigenvalues, counted according to multiplicity, by

$$
0<\mu_{1}\left(\sigma_{1}, \sigma_{2}\right) \leq \mu_{2}\left(\sigma_{1}, \sigma_{2}\right) \leq \ldots \leq \mu_{k}\left(\sigma_{1}, \sigma_{2}\right) \leq \ldots \rightarrow \infty \text { as } k \rightarrow \infty \text {. }
$$

The problem of determining the geometry of $\Omega$ as well as the impedances $\sigma_{1}$ and $\sigma_{2}$ from a complete knowledge of the eigenvalues (1.14) has been discussed by Zayed [21] in the case $n=2$ and by Zayed [22] in the case $n=3$ where $\sigma_{1}$ and $\sigma_{2}$ are positive constants, using the asymptotic expansion of the spectral function

$$
\Theta(t)=\sum_{k=1}^{\infty} \exp \left[-t \mu_{k}\left(\sigma_{1}, \sigma_{2}\right)\right] \text { as } t \rightarrow 0 .
$$

Thus in the case $n=2$, Zayed $[21,23]$ has shown that

$$
\begin{aligned}
\Theta(t)= & \frac{|\Omega|}{4 \pi t}+\frac{\left|\partial \Omega_{1}\right|+\left|\partial \Omega_{2}\right|}{8(\pi t)^{1 / 2}}-\frac{1}{2 \pi}\left(\sigma_{2}\left|\partial \Omega_{2}\right|-\sigma_{1}\left|\partial \Omega_{1}\right|\right) \\
& +\frac{7}{256}\left(\frac{t}{\pi}\right)^{1 / 2} \sum_{i=1}^{2} \int_{\partial \Omega_{i}}\left[K_{i}^{2}(Q)-\frac{64}{7}\left(\frac{\pi \sigma_{i}}{\left|\partial \Omega_{i}\right|}-\sigma_{i}^{2}\right)\right] d Q+0(t) \text { as } t \rightarrow 0,
\end{aligned}
$$

where $\left|\partial \Omega_{1}\right|$ and $K_{1}(Q),\left(Q \varepsilon \partial \Omega_{1}\right)$ are respectively the total length and the curvature of $\partial \Omega_{1}$ while $\left|\partial \Omega_{2}\right|$ and $K_{2}(Q),\left(Q \varepsilon \partial \Omega_{2}\right)$ are respectively the total length and the curvature of $\partial \Omega_{2}$.

In the case $\boldsymbol{n}=3$, Zayed [22] has shown that

$$
\begin{aligned}
\Theta(t)= & \frac{V}{(4 \pi t)^{3 / 2}}+\frac{\left|S_{1}\right|+\left|S_{2}\right|}{16 \pi t}+\frac{1}{12 \pi^{3 / 2} t^{1 / 2}} \sum_{i=1}^{2} \int_{S_{i}}\left[H_{i}(Q)-3 \sigma_{i}\right] d Q \\
& +\frac{7}{128 \pi} \sum_{i=1}^{2} \int_{S_{i}}\left\{\left[H_{i}(Q)-3 \sigma_{i}\right]^{2}-\left[N_{i}(Q)-\frac{26}{7} \sigma_{i} H_{i}(Q)+\frac{47}{7} \sigma_{i}^{2}\right]\right\} d Q+0\left(t^{1 / 2}\right) \text { as } t \rightarrow 0,
\end{aligned}
$$

where $\left|S_{1}\right|, H_{1}(Q)$ and $N_{1}(Q),\left(Q \varepsilon S_{1}\right)$ are respectively the surface area, mean curvature and Gaussian curvature of the surface $S_{1}$, while $\left|S_{2}\right|, H_{2}(Q)$ and $N_{2}(Q),\left(Q \varepsilon S_{2}\right)$ are respectively the surface area, mean curvature and Gaussian curvature of the surface $S_{2}$. Further interpretations of formulae (1.16) and (1.17) can be found in Zayed [21-23].

In Theorem 1, we generalize the results (1.16) and (1.17) to the case when $\sigma_{1}$ and $\sigma_{2}$ are positive functions satisfying the Lipschitz condition, by using the expression

$$
\sum_{k=1}^{\infty}\left\{\mu_{k}\left(\sigma_{1}, \sigma_{2}\right)+P\right\}^{-2} \text {, }
$$

where $P$ is a positive constant.

In Theorem 2, we show that this generalization plays an important role in establishing a method to study the asymptotic behavior of the difference

$$
\sum_{\mu_{k}\left(\sigma_{1}, \sigma_{2}\right) \leq \lambda}\left\{\mu_{k}\left(\alpha_{2}, \beta_{2}\right)-\mu_{k}\left(\alpha_{1}, \beta_{1}\right)\right\}
$$

for large values of $\lambda$, where the three pairs of functions $\left(\sigma_{1}, \sigma_{2}\right),\left(\alpha_{1}, \beta_{1}\right)$ and $\left(\alpha_{2}, \beta_{2}\right)$ are distinct and satisfying the Lipschitz condition and the summation is taken over all values of $k$ for which $\mu_{k}\left(\sigma_{1}, \sigma_{2}\right) \leq \lambda$. The method uses an interesting and important Tauberian theorem due to Hardy and Littlewood and developed by Titchmarsh [24]. 
Theorems 3, 4 and Corollaries 1-5 contain further results which can be considered as a generalization of the results of Theorem 2 .

\section{STATEMENT AND PROOFS OF RESULTS}

THEOREM 1. If the functions $\sigma_{1}(Q), Q \varepsilon \partial \Omega_{1}$ (or $S_{1}$ ) and $\sigma_{2}(Q), Q \varepsilon \partial \Omega_{2}$ (or $S_{2}$ ) satisfy the Lipschitz condition and if $P$ is a positive constant, then in the case $n=2$

$$
\begin{aligned}
\sum_{i=1}^{\infty}\left\{\mu_{k}\left(\sigma_{1}, \sigma_{2}\right)+P\right\}^{-2}= & \frac{|\Omega|}{4 \pi P}+\frac{\left|\partial \Omega_{1}\right|+\left|\partial \Omega_{2}\right|}{16 P^{3 / 2}}-\frac{1}{2 \pi P^{2}}\left\{\int_{\partial \Omega_{2}} \sigma_{2}(Q) d Q-\int_{\partial \Omega_{1}} \sigma_{1}(Q) d Q\right\} \\
& +\frac{21}{1024 P^{5 / 2}} \sum_{t=1}^{2} \int_{\partial \Omega_{t}}\left\{K_{t}^{2}(Q)-\frac{32}{7}\left[\sigma_{\iota}(Q) K_{\iota}(Q)\right.\right. \\
& \left.\left.-2 \sigma_{t}^{2}(Q)\right]\right\} d Q+O\left(\frac{1}{P^{3}}\right) \text { as } P \rightarrow \infty,
\end{aligned}
$$

while in the case $n=3$

$$
\begin{aligned}
\sum_{k=1}^{\infty}\left\{\mu_{k}\left(\sigma_{1}, \sigma_{2}\right)+P\right\}^{-2}= & \frac{V}{8 \pi P^{1 / 2}}+\frac{\left|S_{1}\right|+\left|S_{2}\right|}{16 \pi P}+\frac{1}{24 \pi P^{3 / 2}} \sum_{\imath=1}^{2} \int_{S_{\iota}}\left[H_{\imath}(Q)-3 \sigma_{i}(Q)\right] d Q \\
& +\frac{7}{128 \pi P^{2}} \sum_{1=1}^{2} \int_{S_{t}}\left\{\left[H_{\iota}(Q)-3 \sigma_{\iota}(Q)\right]^{2}-\left[N_{\iota}(Q)\right.\right. \\
& \left.\left.-\frac{26}{7} \sigma_{\iota}(Q) H_{\imath}(Q)+\frac{47}{7} \sigma_{i}^{2}(Q)\right]\right\} d Q+O\left(\frac{1}{P^{5 / 2}}\right) \text { as } P \rightarrow \infty .
\end{aligned}
$$

Note that the expression (1.18) is just the Laplace transform of the function $t \Theta(t)$ with respect to $t$ and $P>0$ is the Laplace transform parameter. With this connection we deduce that formulae (2.1) and (2.2) can be considered as a generalization of formulae (1.16) and (1.17) respectively.

THEOREM 2. If the three pairs of functions $\left(\sigma_{1}(Q), \sigma_{2}(Q)\right),\left(\alpha_{1}(Q), \beta_{1}(Q)\right)$ and $\left(\alpha_{2}(Q), \beta_{2}(Q)\right)$ are distinct and satisfying the Lipschitz condition, then we deduce for $\lambda \rightarrow \infty$ that

$$
\sum_{\mu_{k}\left(\sigma_{1}, \sigma_{2}\right) \leq \lambda}\left\{\mu_{k}\left(\alpha_{2}, \beta_{2}\right)-\mu_{k}\left(\alpha_{1}, \beta_{1}\right)\right\}= \begin{cases}\frac{a_{1}}{1 \pi} \lambda+o(\lambda) & \text { in the case } n=2, \\ \frac{b_{1}}{3 \pi^{2}} \lambda^{3 / 2}+o\left(\lambda^{3 / 2}\right) & \text { in the case } n=3,\end{cases}
$$

where

$$
a_{1}=\int_{\partial \Omega_{2}}\left[\beta_{2}(Q)-\beta_{1}(Q)\right] d Q-\int_{\partial \Omega_{1}}\left[\alpha_{2}(Q)-\alpha_{1}(Q)\right] d Q,
$$

and

$$
b_{1}=\int_{S_{2}}\left[\beta_{2}(Q)-\beta_{1}(Q)\right] d Q+\int_{S_{1}}\left[\alpha_{2}(Q)-\alpha_{1}(Q)\right] d Q .
$$

Formulae (2.3) and (2.4) can be considered as a generalization of the familiar formulae of Gel'fand and Levitan [25] for the difference of traces of two Sturm-Liouville operators.

Let us now give the proofs of Theorems 1,2. To prove Theorem 1, we shall use the Laplace transform of Green's function for the heat equation $\left(\Delta_{n}-\frac{\partial}{\partial t}\right) u=0, n=2$ or 3 with respect to the time $t$, and use $s^{2}$ as the Laplace transform parameter.

PROOF OF THEOREM 1. With reference to [26, Sec. 2], let

$$
\bar{G}\left(\underset{\sim}{x, x_{1}} ;-s^{2}\right)=\frac{1}{2 \pi} K_{0}\left(s\left|\underset{\sim}{x}-\underline{\sim}_{1}\right|\right)-\bar{g}\left(\underset{\sim}{\left.x, x_{1} ;-s^{2}\right)},\right.
$$


be the Green's function of the expression $\left(\Delta_{2}-s^{2}\right) u$ in the domain $\Omega \subseteq R^{2}$ together with the boundary conditions (1.12) and (1.13) on $\partial \Omega_{2}$ respectively, where $s$ is a sufficiently large positive constant while $x$ and $x_{1}$ are points belong to $\Omega$. In (2.5), $K_{0}$ is the modified Bessel function of the second kind and of zero order, while $\bar{g}$ is a regular part of the Green's function.

With reference to [2], we deduce that as $x_{1} \rightarrow x$ the equality

$$
\bar{G}\left(\underset{\sim}{x, x_{1}} ;-s_{1}^{2}\right)-\bar{G}\left(\underset{\sim}{x, x_{1}} ;-s^{2}\right)=\left(s^{2}-s_{1}^{2}\right) \sum_{k=1}^{\infty} \frac{\phi_{k}(\underset{\sim}{x}) \phi_{k}\left(\begin{array}{c}
x_{1} \\
\sim
\end{array}\right)}{\left\{\mu_{k}\left(\sigma_{1}, \sigma_{2}\right)+s_{1}^{2}\right\}\left\{\mu_{k}\left(\sigma_{1}, \sigma_{2}\right)+s^{2}\right\}},
$$

where $\left\{\phi_{k}\left(\begin{array}{l}x \\ \sim\end{array}\right)\right\}$ are normalized eigenfunctions and $s \neq s_{1}$, implies

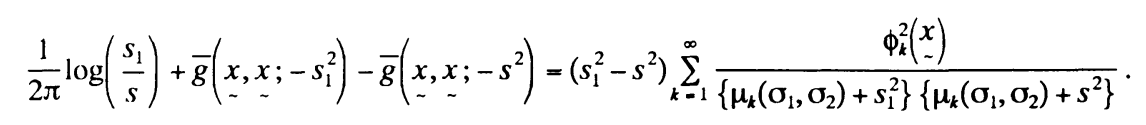

Thus we get the formula

$$
\sum_{k=1}^{\infty}\left\{\mu_{k}\left(\sigma_{1}, \sigma_{2}\right)+s^{2}\right\}^{-2}=\frac{|\Omega|}{4 \pi s^{2}}+\frac{1}{2 s} \iint_{\Omega} \bar{g}_{s}^{\prime}\left(\underset{\sim}{x, x} ;-s^{2}\right) d x .
$$

Using methods similar to those obtained in [14], [21], [23] we can show that

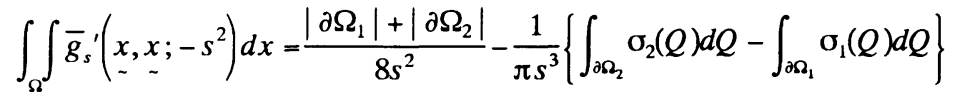

$$
\begin{aligned}
& +\frac{21}{512 s^{4}} \sum_{i=1}^{2} \int_{\partial \Omega_{i}}\left\{K_{i}^{2}(Q)-\frac{32}{7}\left[\sigma_{i}(Q) K_{i}(Q)-2 \sigma_{i}^{2}(Q)\right]\right\} d Q \\
& +0\left(\frac{1}{s^{5}}\right) \text { as } s \rightarrow \infty \text {. }
\end{aligned}
$$

On inserting (2.9) into (2.8) and letting $s^{2}=P$ we arrive at (2.1).

Similarly, let

$$
\bar{G}\left(\underset{\sim}{x, 1} \underset{\sim}{x} ;-s^{2}\right)=\frac{\exp \left(-s\left|\begin{array}{c}
x-x_{1} \\
\sim \\
\sim
\end{array}\right|\right)}{4 \pi\left|\underset{\sim}{x-x_{1}}\right|}-\bar{g}\left(\underset{\sim}{x, x_{1}} ;-s^{2}\right),
$$

be the Green's function of the expression $\left(\Delta_{3}-s^{2}\right) u$ in the domain $\Omega \subseteq R^{3}$ together with the boundary conditions (1.12) and (1.13) on $S_{1}$ and $S_{2}$ respectively.

With reference to [3], we deduce that as $x_{1} \rightarrow x$ the equality (2.6) implies

$$
\frac{\left(s_{1}-s\right)}{4 \pi}+\bar{g}\left(\underset{\sim}{x}, \underset{\sim}{x} ;-s_{1}^{2}\right)-\bar{g}\left(\underset{\sim}{\left.x, x ;-s^{2}\right)}=\left(s_{1}^{2}-s^{2}\right) \sum_{k=1}^{\infty} \frac{\phi_{k}^{2}(\underset{\sim}{x})}{\left\{\mu_{k}\left(\sigma_{1}, \sigma_{2}\right)+s_{1}^{2}\right\}\left\{\mu_{k}\left(\sigma_{1}, \sigma_{2}\right)+s^{2}\right\}} .\right.
$$

Thus we get the formula

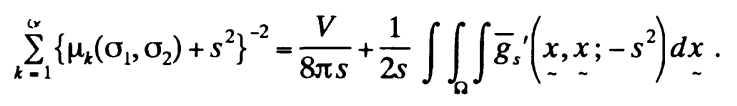

Using methods similar to those obtained in [16], [22], we can show that 


$$
\begin{aligned}
& \iiint_{\Omega} \bar{g}_{s}{ }_{s}\left(\underset{\sim}{x, x} ;-s^{2}\right) d x=\frac{\left|S_{1}\right|+\left|S_{2}\right|}{8 \pi s}+\frac{1}{12 \pi s^{2}} \sum_{1}^{2} \int_{S_{1}}\left[H_{1}(Q)-3 \sigma_{1}(Q)\right] d Q \\
& +\frac{7}{64 \pi s^{3}} \sum_{t=1}^{2} \int_{S_{1}}\left\{\left[H_{l}(Q)-3 \sigma_{\imath}(Q)\right]^{2}-\left[N_{l}(Q)-\frac{26}{7} \sigma_{\imath}(Q) H_{l}(Q)\right.\right. \\
& \left.\left.+\frac{47}{7} \sigma_{i}^{2}(Q)\right]\right\} d Q+0\left(\frac{1}{s^{4}}\right) \text { as } s \rightarrow \infty .
\end{aligned}
$$

On inserting (2.13) into (2.12) and letting $s^{2}=P$ we arrive at (2.2).

Finally, we note that the proof of either (2.9) or (2.13) is omitted here since it is very similar to those obtained in [21] or [22] respectively.

PROOF OF THEOREM 2. With reference to [26], let us assume that $\alpha_{2}(Q) \geq \alpha_{1}(Q),\left(Q \in \partial \Omega_{1}\right)$ and $\beta_{2}(Q) \geq \beta_{1}(Q),\left(Q \in \partial \Omega_{2}\right)$ and introduce the non-negative and non-decreasing function

$$
\left.\Phi(\lambda)=\sum_{\mu_{k}\left(\alpha_{2}, \beta_{2}\right) \leq \lambda}\left\{\mu_{k} \alpha_{2}, \beta_{2}\right)-\mu_{k}\left(\alpha_{1}, \beta_{1}\right)\right\},
$$

moreover we let

$$
\psi(P)=\sum_{k=1}^{\infty} \frac{\left.\left\{\mu_{k}\left(\alpha_{2}, \beta_{2}\right)-\mu_{k}\left(\alpha_{1}, \beta_{1}\right)\right\}^{2}\left\{\mu_{k} \alpha_{2}, \beta_{2}\right)+2 \mu_{k}\left(\alpha_{1}, \beta_{1}\right)+3 P\right\}}{\left\{\mu_{k}\left(\alpha_{2}, \beta_{2}\right)+P\right\}^{3}\left\{\mu_{k}\left(\alpha_{1}, \beta_{1}\right)+P\right\}^{2}} .
$$

Using formula (2.1) first for the functions $\left(\alpha_{1}(Q), \beta_{1}(Q)\right)$, then for the functions $\left(\alpha_{2}(Q), \beta_{2}(Q)\right)$ and subtracting the second one from the first, we find after some reduction that

$$
2 \sum_{k=1}^{\infty} \frac{\left\{\mu_{k}\left(\alpha_{2}, \beta_{2}\right)-\mu_{k}\left(\alpha_{1}, \beta_{1}\right)\right\}}{\left\{\mu_{k}\left(\alpha_{2}, \beta_{2}\right)+P\right\}^{3}}+\psi(P)=\frac{a_{1}}{2 \pi P^{2}}+\frac{3 a_{2}}{16 P^{5 / 2}}+0\left(\frac{1}{P^{3}}\right) \quad \text { as } P \rightarrow \infty,
$$

where

$a_{2}=\int_{\partial \Omega_{2}}\left[\beta_{2}(Q)-\beta_{1}(Q)\right]\left[\frac{1}{2} K_{2}(Q)-\beta_{1}(Q)-\beta_{2}(Q)\right] d Q+\int_{\partial \Omega_{1}}\left[\alpha_{2}(Q)-\alpha_{1}(Q)\right]\left[\frac{1}{2} K_{1}(Q)-\alpha_{1}(Q)-\alpha_{2}(Q)\right] d Q$.

Formula (2.16) can be written for any $c<\mu_{1}\left(\alpha_{2}, \beta_{2}\right)$ in the equivalent form

$2 \int_{c}^{+\infty} \frac{d \Phi(\lambda)}{(\lambda+P)^{3}}+\psi(P)=\frac{a_{1}}{2 \pi P^{2}}+\frac{3 a_{2}}{16 P^{5 / 2}}+0\left(\frac{1}{P^{3}}\right)$ as $P \rightarrow \infty$.

Further, noting that

$$
\psi(P)=o\left\{\int_{c}^{+\infty}(\lambda+P)^{-3} d \Phi(\lambda)\right\} \quad \text { as } P \rightarrow \infty
$$

we get

$$
\int_{c}^{+\infty} \frac{d \Phi(\lambda)}{(\lambda+P)^{3}} \sim \frac{a_{1}}{4 \pi P^{2}} \text { as } P \rightarrow \infty .
$$

Applying a Tauberian Theorem of Hardy and Littlewood (see, for example [24]), we find that

$$
\Phi(\lambda) \sim \frac{a_{1}}{2 \pi} \lambda \text { as } \lambda \rightarrow \infty .
$$

Analogously, one establishes the asymptotic formula

$$
\sum_{\mu_{k}\left(\alpha_{1}, \beta_{1}\right) \leq \lambda}\left\{\mu_{k}\left(\alpha_{2}, \beta_{2}\right)-\mu_{k}\left(\alpha_{1}, \beta_{1}\right)\right\} \sim \frac{a_{1}}{2 \pi} \lambda \text { as } \lambda \rightarrow \infty .
$$

Further, noting that 


$$
\begin{aligned}
\sum_{\mu_{k}\left(\alpha_{2}, \beta_{2}\right)>>\lambda}\left\{\mu_{k}\left(\left(\alpha_{2}, \beta_{2}\right)-\mu_{k}\left(\alpha_{1}, \beta_{1}\right)\right\}\right. & \leq \sum_{\mu_{k}\left(\alpha_{1}, \sigma_{2}\right) \leq \lambda}\left\{\mu_{k}\left(\alpha_{2}, \beta_{2}\right)-\mu_{k}\left(\alpha_{1}, \beta_{1}\right)\right\} \\
& \leq \sum_{\mu_{k}\left(\alpha_{1}, \beta_{1}\right) \leq \lambda}\left\{\mu_{k}\left(\alpha_{2}, \beta_{2}\right)-\mu_{k}\left(\alpha_{1}, \beta_{1}\right)\right\},
\end{aligned}
$$

where

$$
\begin{array}{ll}
\alpha_{1}^{*}(Q)=\min \left\{\sigma_{1}(Q), \alpha_{1}(Q)\right\}, & \beta_{1}^{*}(Q)=\min \left\{\sigma_{2}(Q), \beta_{1}(Q)\right\}, \\
\dot{\alpha}_{2}^{*}(Q)=\min \left\{\sigma_{1}(Q), \alpha_{2}(Q)\right\}, & \beta_{2}^{*}(Q)=\min \left\{\sigma_{2}(Q), \beta_{2}(Q)\right\},
\end{array}
$$

and the fact that as $\lambda \rightarrow \infty$ the functions

$$
\begin{aligned}
\sum_{\mu_{k}\left(\alpha_{2}, \beta_{2}^{*}\right) \leq \lambda}\left\{\mu_{k}\left(\alpha_{2}, \beta_{2}\right)-\mu_{k}\left(\alpha_{1}, \beta_{1}\right)\right\}= & \sum_{\mu_{k}\left(\alpha_{2}, \beta_{2}^{*}\right) \leq \lambda}\left\{\mu_{k}\left(\alpha_{2}^{*}, \beta_{2}^{*}\right)-\mu_{k}\left(\alpha_{1}, \beta_{1}\right)\right\} \\
& -\sum_{\mu_{k}\left(\alpha_{2}, \beta_{2}^{*}\right) \leq \lambda}\left\{\mu_{k}\left(\alpha_{2}^{*}, \beta_{2}^{*}\right)-\mu_{k}\left(\alpha_{2}, \beta_{2}\right)\right\},
\end{aligned}
$$

and likewise for $\left(\alpha_{1}^{*}, \beta_{1}^{*}\right)$ are asymptotically equal to $\frac{a_{1}}{2 \pi} \lambda$, we obtain $(2.3)$ for the special case $\alpha_{2}(Q) \geq \alpha_{1}(Q),\left(Q \in \partial \Omega_{1}\right)$ and $\beta_{2}(Q) \geq \beta_{1}(Q),\left(Q \in \partial \Omega_{2}\right)$.

Similarly, we derive (2.4) for the special case $\alpha_{2}(Q) \geq \alpha_{1}(Q), \quad\left(Q \in S_{1}\right)$ and $\beta_{2}(Q) \geq \beta_{1}(Q),\left(Q \in S_{2}\right)$ as follows: Using formula (2.2) first for the functions $\left(\alpha_{1}(Q), \beta_{1}(Q)\right)$, then for the functions $\left(\alpha_{2}(Q), \beta_{2}(Q)\right)$ and subtracting the second one from the first, we find for any $c<\mu_{1}\left(\alpha_{2}, \beta_{2}\right)$ that

$$
2 \int_{c}^{+\infty} \frac{d \Phi(\lambda)}{(\lambda+P)^{3}}+\psi(P)=\frac{b_{1}}{8 \pi P^{3 / 2}}+\frac{b_{2}}{8 \pi P^{2}}+0\left(\frac{1}{P^{5 / 2}}\right) \text { as } P \rightarrow \infty,
$$

where

$$
b_{2}=\int_{S_{2}}\left[\beta_{2}(Q)-\beta_{1}(Q)\right]\left[H_{2}(Q)-\beta_{1}(Q)-\beta_{2}(Q)\right] d Q+\int_{S_{1}}\left[\alpha_{2}(Q)-\alpha_{1}(Q)\right]\left[H_{1}(Q)-\alpha_{1}(Q)-\alpha_{2}(Q)\right] d Q .
$$

On using the same nature of $\psi(P)$, we write the integral in (2.23) in the asymptotic form

$$
\int_{c}^{+\infty} \frac{d \Phi(\lambda)}{(\lambda+P)^{3}} \sim \frac{b_{1}}{16 \pi P^{3 / 2}} \text { as } P \rightarrow \infty .
$$

Consequently, we deduce that

$$
\Phi(\lambda) \sim \frac{b_{1}}{3 \pi^{2}} \lambda^{3 / 2} \text { as } \lambda \rightarrow \infty .
$$

Analogously, one establishes the asymptotic formula

$$
\sum_{\mu_{k}\left(\alpha_{1}, \beta_{1}\right) \leq \lambda}\left\{\mu_{k}\left(\alpha_{2}, \beta_{2}\right)-\mu_{k}\left(\alpha_{1}, \beta_{1}\right)\right\} \sim \frac{b_{1}}{3 \pi^{2}} \lambda^{3 / 2} \text { as } \lambda \rightarrow \infty .
$$

On using (2.21) and the fact that as $\lambda \rightarrow \infty$ the functions (2.22) for $\left(\alpha_{2}^{*}, \beta_{2}^{*}\right)$ and likewise for $\left(\alpha_{1}^{*}, \beta_{1}^{*}\right)$ are asymptotically equal to $\frac{b_{1}}{3 \pi^{2}} \lambda^{3 / 2}$, we obtain (2.4) for the special case $\alpha_{2}(Q) \geq \alpha_{1}(Q),\left(Q \in S_{1}\right)$ and $\beta_{2}(Q) \geq \beta_{1}(Q),\left(Q \in S_{2}\right)$.

In order to prove the theorem in the general case it is sufficient to apply the equality

$$
\begin{aligned}
\sum_{\mu_{k}\left(\sigma_{1}, \sigma_{2}\right) \leq \lambda}\left\{\mu_{k}\left(\alpha_{2}, \beta_{2}\right)-\mu_{k}\left(\alpha_{1}, \beta_{1}\right)\right\}= & \sum_{\mu_{k}\left(\sigma_{1}, \sigma_{2}\right) \leq \lambda}\left\{\mu_{k}\left(\sigma_{0}, \sigma_{0}^{*}\right)-\mu_{k}\left(\alpha_{1}, \beta_{1}\right)\right\} \\
& -\sum_{\mu_{k}\left(\sigma_{1}, \sigma_{2}\right) \leq \lambda}\left\{\mu_{k}\left(\sigma_{0}, \sigma_{0}^{*}\right)-\mu_{k}\left(\alpha_{2}, \beta_{2}\right)\right\},
\end{aligned}
$$

where 


$$
\sigma_{0}(Q)=\max \left\{\alpha_{1}(Q), \alpha_{2}(Q)\right\}, \quad \sigma_{0}^{*}(Q)=\max \left\{\beta_{1}(Q), \beta_{2}(Q)\right\},
$$

and apply the special case of the theorem which we just proved.

\section{FURTHER RESULTS}

COROLLARY 1. On using formulae (1.4) and (1.5) we deduce as $m \rightarrow \infty$ that

$$
\sum_{k=1}^{m}\left\{\mu_{k}\left(\alpha_{2}, \beta_{2}\right)-\mu_{k}\left(\alpha_{1}, \beta_{1}\right)\right\}= \begin{cases}\left(\frac{2 a_{1}}{|\Omega|}\right) m+o(m) & \text { in the case } n=2, \\ \left(\frac{2 b_{1}}{V}\right) m+o(m) & \text { in the case } n=3 .\end{cases}
$$

Using Theorem 2 we easily prove the following Theorems:

THEOREM 3. Let the three pairs of functions $\left(\sigma_{1}(Q), \sigma_{2}(Q)\right),\left(\alpha_{1}(Q), \beta_{1}(Q)\right),\left(\alpha_{2}(Q), \beta_{2}(Q)\right)$ and the quantity $a_{1} \neq 0$ be the same as in (2.3). Furthermore, on the half-axis $[c,+\infty)$ let a function $f(\lambda)$ of constant sign be given which is absolutely continuous on each interval $[c, d], d<\infty$; further we assume that the expression $\frac{\lambda f^{(\lambda)}}{f(\lambda)}$ is bounded almost everywhere and $\int_{c}^{+\infty} f(\lambda) d \lambda=\infty$. Then as $\lambda \rightarrow \infty$ we get

$$
\sum_{0<\mu_{k}\left(\sigma_{1}, \sigma_{2}\right) \leq \lambda} f\left[\mu_{k}\left(\sigma_{1}, \sigma_{2}\right)\right]\left\{\mu_{k}\left(\alpha_{2}, \beta_{2}\right)-\mu_{k}\left(\alpha_{1}, \beta_{1}\right)\right\}=\left[\frac{a_{1}}{2 \pi}+o(1)\right] \int_{c}^{\lambda} f(t) d t .
$$

THEOREM 4. Let the three pairs of functions $\left(\sigma_{1}(Q), \sigma_{2}(Q)\right),\left(\alpha_{1}(Q), \beta_{1}(Q)\right),\left(\alpha_{2}(Q), \beta_{2}(Q)\right)$ and the quantity $b_{1} \neq 0$ be the same as in (2.4). Furthermore, on the half-axis $[c,+\infty)$ let a function $f(\lambda)$ of constant sign be given which is absolutely continuous on each interval $[c, d], d<\infty$, further we assume that the expression $\frac{\lambda f(\lambda)}{f(\lambda)}$ is bounded almost everywhere and $\int_{c}^{+\infty} \lambda^{1 / 2} f(\lambda) d \lambda=\infty$. Then as $\lambda \rightarrow \infty$ we get

$$
\sum_{0<\mu_{k}\left(\sigma_{1}, \sigma_{2}\right) \leq \lambda} f\left[\mu_{k}\left(\sigma_{1}, \sigma_{2}\right)\right]\left\{\mu_{k}\left(\alpha_{2}, \beta_{2}\right)-\mu_{k}\left(\alpha_{1}, \beta_{1}\right)\right\}=\left[\frac{b_{1}}{2 \pi^{2}}+o(1)\right] \int_{c}^{\lambda}|t|^{1 / 2} f(t) d t .
$$

PROOF. On setting

$$
Z(\lambda)=\sum_{0<\mu_{k}\left(\sigma_{1}, \sigma_{2}\right) \leq \lambda}\left\{\mu_{k}\left(\alpha_{2}, \beta_{2}\right)-\mu_{k}\left(\alpha_{1}, \beta_{1}\right)\right\}
$$

where the summation is taken over all values of $k$, for which $\mu_{k}\left(\sigma_{1}, \sigma_{2}\right) \leq \lambda$, we deduce for any $c<\mu_{1}\left(\sigma_{1}, \sigma_{2}\right)$ that

$$
\sum_{0<\mu_{k}\left(\sigma_{1}, \sigma_{2}\right) \leq \lambda} f\left[\mu_{k}\left(\sigma_{1}, \sigma_{2}\right)\right]\left\{\mu_{k}\left(\alpha_{2}, \beta_{2}\right)-\mu_{k}\left(\alpha_{1}, \beta_{1}\right)\right\}=\int_{c}^{+\infty} f(\lambda) d Z(\lambda) .
$$

On inserting (2.3) and (2.4) into (3.5) we get easily (3.3) and (3.4) respectively.

COROLLARY 2. On using the mean value theorem, we deduce for any $c<\mu_{1}\left(\sigma_{1}, \sigma_{2}\right)$ that

$$
\begin{aligned}
& \sum_{k=1}^{\infty}\left\{f\left[\mu_{k}\left(\sigma_{2}, \beta_{2}\right)\right]-f\left[\mu_{k}\left(\alpha_{1}, \beta_{1}\right)\right]\right\}= \\
& \sum_{0<\mu_{k}\left(\sigma_{1}, \sigma_{2}\right) \leq \lambda} f^{\prime}\left[\mu_{k}\left(\sigma_{1}, \sigma_{2}\right)\right]\left\{\mu_{k}\left(\alpha_{2}, \beta_{2}\right)-\mu_{k}\left(\alpha_{1}, \beta_{1}\right)\right\}=\int_{c}^{+\infty} f^{\prime}(\lambda) d Z(\lambda),
\end{aligned}
$$

where $\mu_{k}\left(\alpha_{1}, \beta_{1}\right) \leq \mu_{k}\left(\sigma_{1}, \sigma_{2}\right) \leq \mu_{k}\left(\alpha_{2}, \beta_{2}\right)$ and the summation is taken over all values of $k$, for which $\mu_{k}\left(\sigma_{1}, \sigma_{2}\right) \leq \lambda$.

Consequently, if $f(\lambda)=\lambda^{i}, i>0$ we deduce for $m \rightarrow \infty$ that in the case $n=2$ 


$$
\sum_{k=1}^{m}\left\{\mu_{k}^{i}\left(\alpha_{2}, \beta_{2}\right)-\mu_{k}^{i}\left(\alpha_{1}, \beta_{1}\right)\right\}=\frac{a_{1}}{2 \pi}\left(\frac{4 \pi}{|\Omega|}\right)^{\prime} m^{i}+o\left(m^{i}\right),
$$

while if $f(\lambda)=\lambda^{\prime}, i \geq-\frac{1}{2}$ we deduce for $m \rightarrow \infty$ that in the case $n=3$

$$
\sum_{k=1}^{m}\left\{\mu_{k}^{i}\left(\alpha_{2}, \beta_{2}\right)-\mu_{k}^{i}\left(\alpha_{1}, \beta_{1}\right)\right\}= \begin{cases}\frac{i b_{1}}{\pi^{2}(2 i+1)}\left(\frac{6 \pi^{2}}{V} m\right)^{(2+1) / 3}+o\left(m^{2+1) 3}\right) & \text { if } i>-\frac{1}{2} \\ \frac{-b_{1}}{6 \pi^{2}} \ln \left(\frac{6 \pi^{2}}{V} m\right)+o\left(\ln \left(\frac{6 \pi^{2}}{v} m\right)\right) & \text { if } i=-\frac{1}{2} .\end{cases}
$$

COROLLARY 3. Assuming that the function $f(\lambda)$ of Theorem 3 has the form: $f(\lambda)=\lambda^{i}, i \geq-1$ then we deduce as $\lambda \rightarrow \infty$ that

$$
\sum_{0<\mu_{k}\left(\sigma_{1}, \sigma_{2}\right) \leq \lambda} \mu_{k}^{i}\left(\sigma_{1}, \sigma_{2}\right)\left\{\mu_{k}\left(\alpha_{2}, \beta_{2}\right)-\mu_{k}\left(\alpha_{1}, \beta_{1}\right)\right\}= \begin{cases}\frac{a_{1}}{2 \pi(i+1)} \lambda^{i+1}+o\left(\lambda^{i+1}\right) & \text { if } i>-1, \\ \frac{a_{1}}{2 \pi} \ln \lambda+o(\ln \lambda) & \text { if } i=-1 .\end{cases}
$$

COROLLARY 4. Assuming that the function $f(\lambda)$ of Theorem 4 has the form $f(\lambda)=\lambda^{i}, i \geq-3 / 2$ we deduce for $\lambda \rightarrow \infty$ that

$$
\sum_{0<\mu_{k}\left(\sigma_{1}, \sigma_{2}\right) \leqslant \lambda} \mu_{k}^{i}\left(\sigma_{1}, \sigma_{2}\right)\left\{\mu_{k}\left(\alpha_{2}, \beta_{2}\right)-\mu_{k}\left(\alpha_{1}, \beta_{1}\right)\right\}= \begin{cases}\frac{b_{1}}{\pi^{2}(2 i+3)} \lambda^{(2 i+3) / 2}+o\left(\lambda^{(2 i+3) / 2}\right) & \text { if } i>-3 / 2 \\ \frac{b_{1}}{2 \pi^{2}} \ln \lambda+o(\ln \lambda) & \text { if } i=-3 / 2 .\end{cases}
$$

COROLLARY 5. If $\mu_{k}\left(\alpha_{1}, \beta_{1}\right) \neq 0$ we deduce for $m \rightarrow \infty$ that in the case $n=2$

$$
\sum_{k=1}^{m} \frac{\mu_{k}\left(\alpha_{2}, \beta_{2}\right)}{\mu_{k}\left(\alpha_{1}, \beta_{1}\right)}=m+\frac{a_{1}}{2 \pi} \ln \left(\frac{4 \pi}{|\Omega|} m\right)+o\left(\ln \left(\frac{4 \pi}{|\Omega|} m\right)\right),
$$

while in the case $n=3$

$$
\sum_{k=1}^{m} \frac{\mu_{k}\left(\alpha_{2}, \beta_{2}\right)}{\mu_{k}\left(\alpha_{1}, \beta_{1}\right)}=m+b_{1}\left(\frac{6}{\pi^{4} V}\right)^{1 / 3} m^{1 / 3}+o\left(m^{1 / 3}\right)
$$

\section{REFERENCES}

[1] COURANT, R. and HILBERT, D., Methods of Mathematical Physics, Vol. 1, WileyInterscience, New York, 1953.

[2] PLEIJEL, Å., A study of certain Green's functions with applications in the theory of vibrating membranes, Ark. Mat. 2 (1954), 553-569.

[3] , On Green's functions and the eigenvalue distribution of the three-dimensional membrane equation, Skand. Mat. Konger. XII, (1954), 222-240.

[4] KAC, M., Can one hear the shape of a drum? Amer. Math. Monthly, 73 (1966), 1-23.

[5] McKEAN, H. P., Jr. and SINGER, I. M., Curvature and the eigenvalues of the Laplacian, J. Diff. Geom, 1 (1967), 43-69.

[6] STEWARTSON, K. and WAECHTER, R. T., On hearing the shape of a drum: further results, Proc. Camb. Philos. Soc., 69 (1971), 353-363.

[7] WAECHTER, R. T., On hearing the shape of a drum: An extension to higher dimensions, Proc. Camb. Philos. Soc., 72 (1972), 439-447.

[8] GREINER, P., An asymptotic expansion for the heat equation, Arch. Rat. Mech. Anal., 41 (1971), 163-218.

[9] SMITH, L., The asymptotics of the heat equation for a boundary value problem, Invent. Math. 63 (1981), 467-493. 
[10] GOTTLIEB, H. P., Hearing the shape of an annular drum, J. Austral. Math. Soc. Ser. B 24 (1983), 435-438.

[11] Eigenvalues of the Laplacian with Neumann boundary conditions, J. Austral. Math. Soc. Ser. B 26 (1985), 293-309.

[12] Eigenvalues of the Laplacian for rectilinear regions, J. Austral. Math. Soc. Ser. B 29(1988), 270-281.

[13] HSU, P., On the $\Theta$-function of a compact Riemannian manifold with boundary, C. R. Acad. Sci. Paris, 309 (1989), 507-510.

[14] SLEEMAN, B. D. and ZAYED, E. M. E., An inverse eigenvalue problem for a general convex domain, J. Math. Anal. Appl., 94 (1983), 78-95.

[15] Trace formulae for the eigenvalues of the Laplacian, J. Appl. Math. Phys. (ZAMP), 35 (1984), 106-115.

[16] ZAYED, E. M.E., An inverse eigenvalue problem for a general convex domain: An extension to higher dimensions, J. Math. Anal. Appl. 112 (1985), 455-470.

[17] Eigenvalues of the Laplacian: An extension to higher dimensions, IMA J. Appl. Math., 33 (1984), 83-99.

[18] Eigenvalues of the Laplacian for the third boundary value problem, J. Austral. Math. Soc. Ser. B 29 (1987), 79-87.

[19] Eigenvalues of the Laplacian for the third boundary value problem: An extension to higher dimensions, J. Math. Anal. Appl., 130 (1988), 78-96.

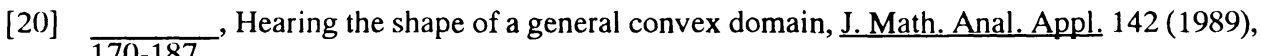

[21] On hearing the shape of an arbitrary doubly-connected region in $R^{2}$, J. Austral. Math. Soc. Ser. B 31 (1990), 472-483.

[22] Hearing the shape of a general doubly-connected domain in $R^{3}$ with impedance boundary conditions, J. Math. Phys., 31 (1990), 2361-2365.

[23] Heat equation for an arbitrary multiply-connected region in $R^{2}$ with impedance boundary conditions, IMA J. Appl. Math., 45 (1990), 233-241.

[24] TITCHAMRSH, E. C., Eigenfunction Expansions Associated with Second Order Differential Equations, Vol. 2, Clarendon Press, Oxford, 1958.

[25] GEL'FAND, I. M. and LEVITAN, B. M., On a simple identity for eigenvalues of a second order differential operator, Dokl. Akad. Nauk. SSSR 88 (1953), 593-596.

[26] ZAYED, E. M. E., Some asymptotic spectral formulae for the eigenvalues of the Laplacian, J. Austral. Math. Soc. Ser. B 30 (1988), 220-229. 


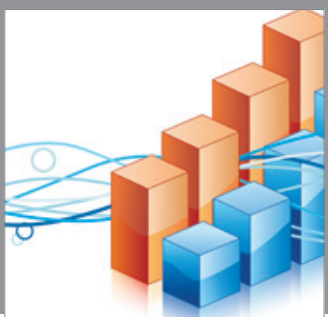

Advances in

Operations Research

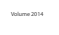

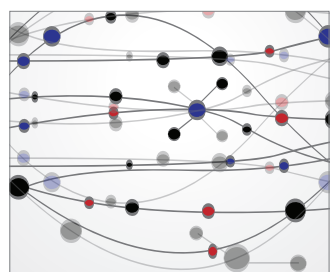

\section{The Scientific} World Journal
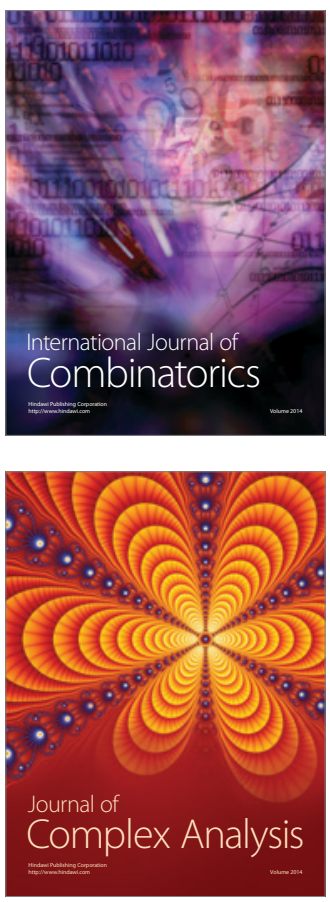

International Journal of

Mathematics and

Mathematical

Sciences
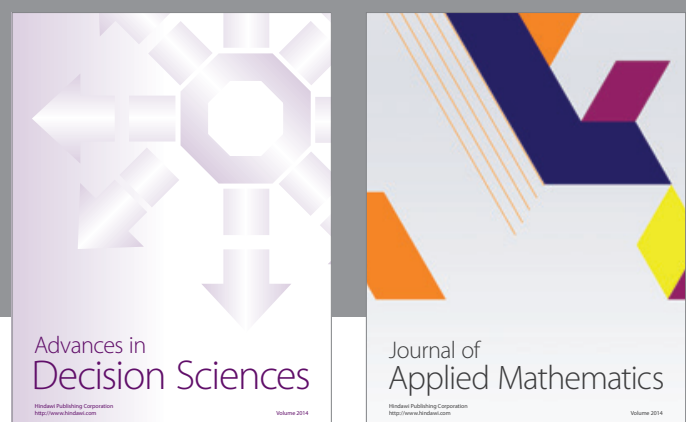

Journal of

Applied Mathematics
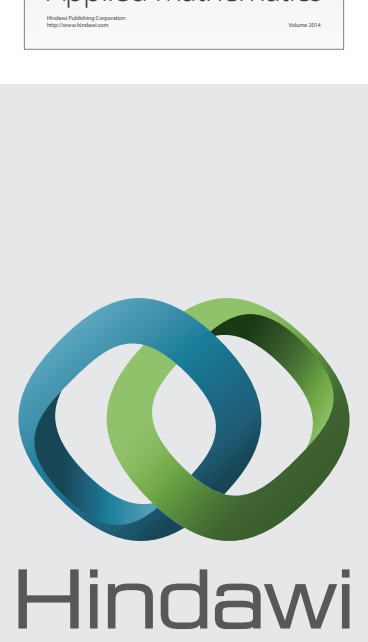

Submit your manuscripts at http://www.hindawi.com
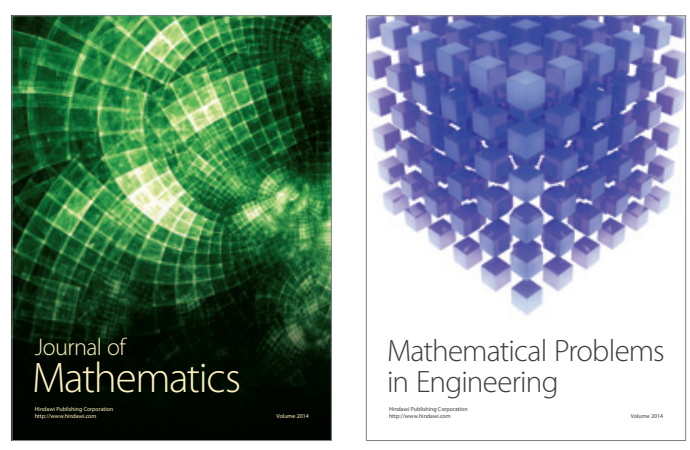

Mathematical Problems in Engineering
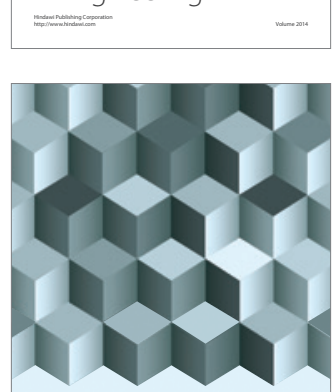

Journal of

Function Spaces
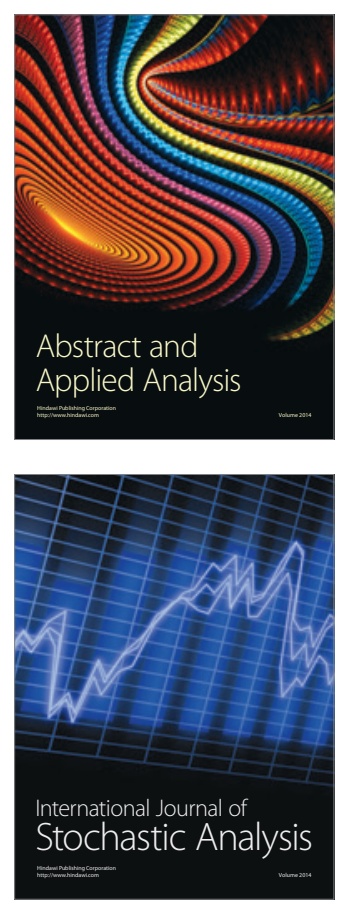

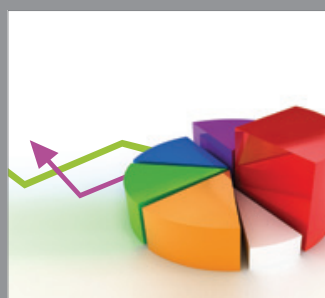

ournal of

Probability and Statistics

Promensencen
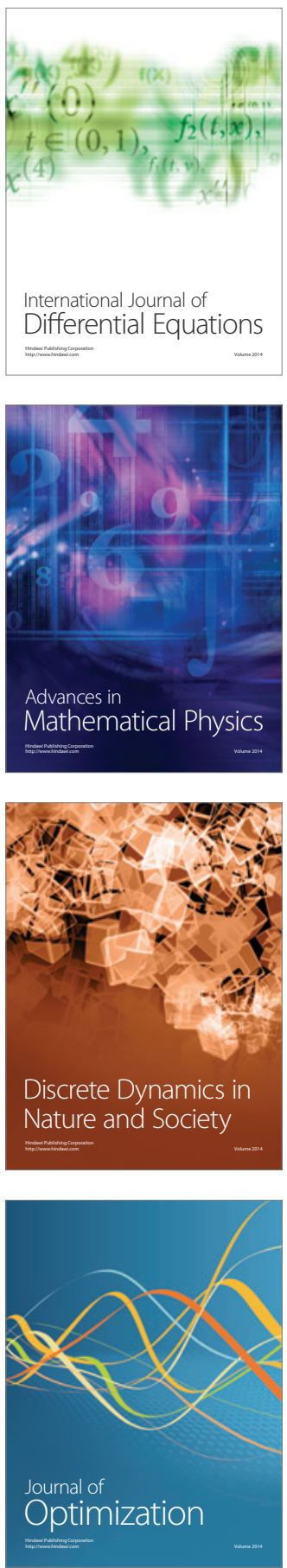